\title{
Chirality-induced polarization effects in the cuticle of scarab beetles: 100 years after Michelson
}

\author{
Hans Arwin, Roger Magnusson, Jan Landin and Kenneth Järrendahl
}

\section{Linköping University Post Print}

N.B.: When citing this work, cite the original article.

This is an electronic version of an article published in:

Hans Arwin, Roger Magnusson, Jan Landin and Kenneth Järrendahl, Chirality-induced polarization effects in the cuticle of scarab beetles: 100 years after Michelson, 2012, Philosophical Magazine, (92), 12, 1583-1599.

Philosophical Magazine is available online at informaworldTM:

http://dx.doi.org/10.1080/14786435.2011.648228

Copyright: Taylor \& Francis: STM, Behavioural Science and Public Health Titles / Taylor \& Francis

http://www.tandf.co.uk/journals/default.asp

Postprint available at: Linköping University Electronic Press

http://urn.kb.se/resolve?urn=urn:nbn:se:liu:diva-77876 


\title{
RESEARCH ARTICLE
}

\section{Chirality-induced polarization effects in the cuticle of scarab beetles: 100 years after Michelson}

\author{
Hans Arwin*, Roger Magnusson, Jan Landin and Kenneth Järrendahl \\ Department of Physics, Chemistry and Biology, Linköping University, SE-581 83 \\ Linköping, Sweden \\ (Received 00 Month 200x; final version received 00 Month 200x)
}

\begin{abstract}
One hundred years ago Michelson discovered circular polarization in reflection from beetles. Today a novel Mueller-matrix ellipsometry setup allows unprecedented detailed characterization of the beetles' polarization properties. A formalism based on elliptical polarization for description of reflection from scarab beetles is here proposed and examples are given on four beetles of different character: Coptomia laevis - a simple dielectric mirror; Cetonia aurata - a left-hand narrow-band elliptical polarizer; Anoplognathus aureus - a broad-band elliptical polarizer; and Chrysina argenteola - a left-hand polarizer for visible light at small angles whereas for larger angles, red reflected light is right-handed polarized. We confirm the conclusion of previous studies which showed that a detailed quantification of ellipticity and degree of polarization of cuticle reflection can be performed instead of only determining whether reflections are circularly polarized or not. We additionally investigate reflection as a function of incidence angle. This provides much richer information for understanding behaviour of beetles and for structural analysis.
\end{abstract}

Keywords: Scarab beetles; Mueller-matrix ellipsometry; elliptical polarization; structural colors

\section{Introduction}

The first observation of chirality in reflection of light from beetles is accredited to Michelson who 100 years ago (April 1911) published the paper "On Metallic Colouring in Birds and Insects" in Philosophical Magazine [1]. Michelson used a Babinet compensator to study reflection from the scarab beetle Plusiotis (now Chrysina) resplendens (Boucard, 1875) and reported "... the reflected light was circularly polarized even at normal incidence...". Michelson described the beetle as it "appears as if coated with an electrolytic deposit of metal, with a lustre resembling brass". Furthermore he concluded that the effect must be due to a "screw structure" of ultra microscopic dimensions. Robinson [2] and later Neville and Caveney [3] discussed these effects as analogues of cholesteric liquid crystals. Bouligand [4] proposed that a lamellated twisted structure causes the observed effects. More recently several reviews of the structural origin of such effects are found [5-8]. A comprehensive survey has been performed by Pye [9] who investigated more than 19000 species of scarab beetles with circularly polarizing filters. In addition to the already known five subfamilies of Scarabaeidae, he found three more subfamilies of Scarabaeidae and one subfamily in the family Hybosoridae exhibiting circular polarization. Recent reports on the taxonomy of the Scarabaeoidea superfamily make it plausible to reduce these nine subfamilies to six or seven $[10,11]$.

*Corresponding author. E-mail: han@ifm.liu.se

ISSN: 1478-6435 print/ISSN 1478-6443 online

(C) 200x Taylor \& Francis

DOI: $10.1080 / 14786435.20 x x \cdot \operatorname{xxxxxx}$

http://www.informaworld.com 

limited to classification in circular or not circular polarization ${ }^{1}$. The spectral variation in polarization is rarely reported and similar holds for angle of incidence dependence. For instance, Pye recently [9] introduced a quantification of the strength of polarization through a parameter $\alpha$ equal to the ratio of irradiances measured using left-hand circularly and right-hand circularly polarized filters. $\alpha$ is asymmetric with respect to handedness and has values in the range 0 to 1 for right-handed circularly polarized light, is equal to 1 for unpolarized light and is larger than 1 for left-handed circularly polarized light. $\alpha$ provides a means for a quantitative comparison among beetles and for measurements on different parts on one and the same beetle but is an incomplete parameter from an optical point of view as only circular effects are included. This is obvious as, for example, totally linearly polarized light would have $\alpha=1$, i.e. the same as for unpolarized light. Furthermore, $\alpha$ will depend on the ellipticity of elliptically polarized light. For a better understanding of the polarization phenomena in scarab beetles there is thus a need for a more precise parameterization of the reflected light including

- a generalization from circular to elliptical polarization;

- a well-defined degree of polarization parameter; and

- quantification of the wavelength and angle of incidence dependence of polarization features.

In particular it is clear that right-handed polarization should not be treated as a rarely occurring special case as often is done, e.g. by Pye who says that this only occurs in single individuals of Chrysina resplendens [9]. In fact we show that the same beetle can exhibit both left- and right-handed effects but at different wavelengths and, remarkably also at the same wavelength but at different angles of incidence.

Many optical studies of reflection properties of natural nanostructures have been performed but modeling of these structures is generally rather rudimentary [12]. One early example is given by Caveney [13] who presented a structural model for the left- as well as right-handed polarization effects in Chrysina resplendens. As a further example, Lowrey et al. [14] studied light reflected from scarab beetles illuminated with unpolarized light. They observed that the light is composed of a sum of unpolarized and elliptically polarized light. A chirped stack of chiral resonators was proposed as a model describing these reflection properties. The same group has also studied structural colors in the green and red manuka beetle Pyronota festiva (Fabricius, 1775) from New Zealand. In addition, they nanoengineered chiral reflectors in titanium oxide with polarization properties and colors resembling those of the beetles [15].

An important step forward is the work by Hodgkinson et al. [16]. They measured full Mueller-matrix spectra with a low-cost ellipsometer at near-normal incidence ( $15^{\circ}$ from normal) on four different species of beetles, other species than ours. Simulations were performed to explain the various polarization properties found and depolarization was addressed. One of the beetles, Chrysina resplendens, was found to be more complex and an ensemble of laterally incoherent chiral thin film reflectors was necessary to include to simulate the data.

\footnotetext{
${ }^{1}$ In many reports discussing polarizing phenomena in scarab beetles, the term "circular polarization" is not well defined and often refers to the phenomenon that a beetle appears to have different color when viewed through a left-hand and a right-hand polarizer. We will here use a more strict definition and use the term "circular polarization" only when the polarization ellipse is circular as further detailed in the theory section.
} 

ther examples are found in the review by Lenau and Barfoed [6]. It has also been demonstrated that reflection from wing scales of Morpho butterflies are different in different vapours indicating that sensor applications are feasible [18]. Focused ion beam chemical vapor deposition was used by Watanabe and coworkers to fabricate replicas of wing scales from Morpho butterflies which display brilliant blue colors [19]. Parker and Townley have discussed that not only conventional engineering methods can be used to develop biomimetic applications but that also mimicking nanoengineering in living cells is a route to make nanostructures with commercial applications [20].

Although phenomenological studies and simulation of the optical response of natural photonic structures are of great value, they are not sufficient in an analytic approach with the objective to extract structural and optical parameters from biological samples. Examples of such parameters are layer thicknesses in multilayered structures, pitch (period) of helicoidal structures, distribution and gradients of thicknesses and the spectral variations of the refractive index and birefringence of the materials constituting the structures. Simple reflectance measurements are not sufficient for retrieving such details. Polarimetric studies adds the polarization dimension and, as shown by Hegedüs et al., imaging polarimetry applied to scarab beetles can be very powerful for mapping out polarization patterns [21]. Natural photonic structures are generally hard to investigate quantitatively by optical methods as they, from an optics point of view, are non-ideal, having surface curvature, scattering phenomena, lateral and in-depth inhomogeneities and anisotropy. Furthermore the optical functions of the constituent materials are not known with sufficient precision or at all.

For resolving optical and structural properties in detail, a more powerful method than reflectometry is ellipsometry [22]. Ellipsometry is based on analyzing the change in polarization upon reflection which provides more information than reflectivity. To make use of the potential of ellipsometry, spectroscopy should be performed. However, ellipsometry has rarely been used on natural nanostructures. Early work by Brink and Lee [23] demonstrated that optical constants of scale material on wings of insects may be determined using null ellipsometry.

In addition to polarization changes, the natural structures may cause changes in the degree of polarization of the reflected light. In nature the incident light is mainly unpolarized but reflected light from an insect can be highly polarized. Conversely, depolarization may occur and light may be partly depolarized due to reflection. The depolarization power of an insect cuticle is thus an important parameter to quantify and it carries information about the cuticle structure.

Due to recent development in ellipsometric instrumentation and methodology, so called Mueller-matrix ellipsometry is now available and can be used to address polarization phenomena as well as nanostructural properties. A Mueller-matrix description of a surface contains the complete linear optical response in terms of irradiance reflectance and polarization properties including depolarization effects. Goldstein used a normal incidence reflectometer to determine Mueller matrices of the scarab beetles Plusiotis clypealis, Plusiotis gloriosa and Plusiotis resplendens in the spectral range 400-700 $\mathrm{nm}$ [24]. In Goldstein's pioneering work it is concluded that the sign of the Mueller-matrix element $m_{41}$ (denoted $M_{30}$ in reference [24]) shows that Plusiotis clypealis and Plusiotis gloriosa provide left-handed polarized light whereas Plusiotis resplendens can provide either left-handed or right-handed polarized light depending on wavelength. The reflected light is not completely circularly polarized but is near-circularly polarized with an ellipticity of up to 0.8 . 
Total circular polarization would require that the matrix elements $M_{10}$ and $M_{20}$ in reference [24] both are zero.

The work of Goldstein experimentally settles the debated question whether there are only left-hand polarizing structures found in beetles or if there also are righthand polarizing structures. Even though right-handedness was discussed already by Michelson it has not been quantified clearly. In the comprehensive review by Pye [9], right-handed polarization is for example only briefly mentioned and not addressed quantitatively at all. It should here be mentioned to credit Michelson that he found that in the red end of the spectrum ".. polarization in the opposite sense appear..." and that Neville and Caveney later claimed that Michelson was wrong and had presented a misleading result [3]. In our opinion, the current picture is too simplified and hampers the development in the field. We claim that it is not only handedness but also the ellipticity of the light and degree of polarization which are relevant as will be addressed here by analyzing spectral Mueller-matrix ellipsometry data. The angle of incidence dependence is also in most cases left out of the discussion. Goldstein [24], for example, only studied normal incidence properties.

Our objective is to generalize the description of polarization of light reflected from natural photonic structures, applied to scarab beetles. Specifically we bring forward, as also was done in Ref. [16], that the issue is not limited to whether beetles reflect left- or right-handed circular polarization but rather to quantify the polarization state (azimuth, ellipticity, handedness) as well as the degree of polarization of the reflected light. We demonstrate that spectral and angle-of-incidence dependences of these parameters can be measured with very high precision using Mueller-matrix ellipsometry. Such data will be very valuable for future detailed analysis of cuticle structure and will allow extraction of structural and optical parameters. It will also help to understand the role of polarization in biology.

Similar work performed at near-normal incidence by Hodgkinson et al. [16] has proved the applicability of Mueller-matrix ellipsometry for studies of beetles. They also demonstrated that simulations based on thin film interference models can provide a qualitative description of structure. However, to the authors knowledge, angular-resolved Mueller-matrix ellipsometry is here for the first time employed in a detailed spectroscopic study of beetle cuticles. Additional advances are that a spot size below $100 \mu \mathrm{m}$ is used and that true ellipsometric data acquisition are employed to record Mueller matrices instead of consecutive reflectance measurements. These features improve data quality and availability of high-precision data will facilitate the use of regression methods for analysis instead of more simple simulation approaches.

\section{Beetle specimens}

Specimens of Chrysina argenteola (Bates, 1888), Coptomia laevis (Waterhouse, 1879) and Anoplognathus aureus (Waterhouse, 1889) were loans from Museum of Natural History in Stockholm. Specimens of Cetonia aurata (Linnaeus, 1758) were collected locally by one of the authors (J.L.). The pinned beetles, shown in Figure 1, were mounted and aligned in the ellipsometer without any treatment. The four species were selected due to their different reflection characteristics. By using simple eye glasses with one left-polarizing and one right-polarizing filter (like in eye glasses for 3D movies), the partly green and partly blue Coptomia laevis displays no difference between viewing through left- or right-hand polarizing filters. The green Cetonia aurata becomes almost black with a right-hand filter and virtually no difference compared to the naked eye is seen with a left-hand polarizing filter. 


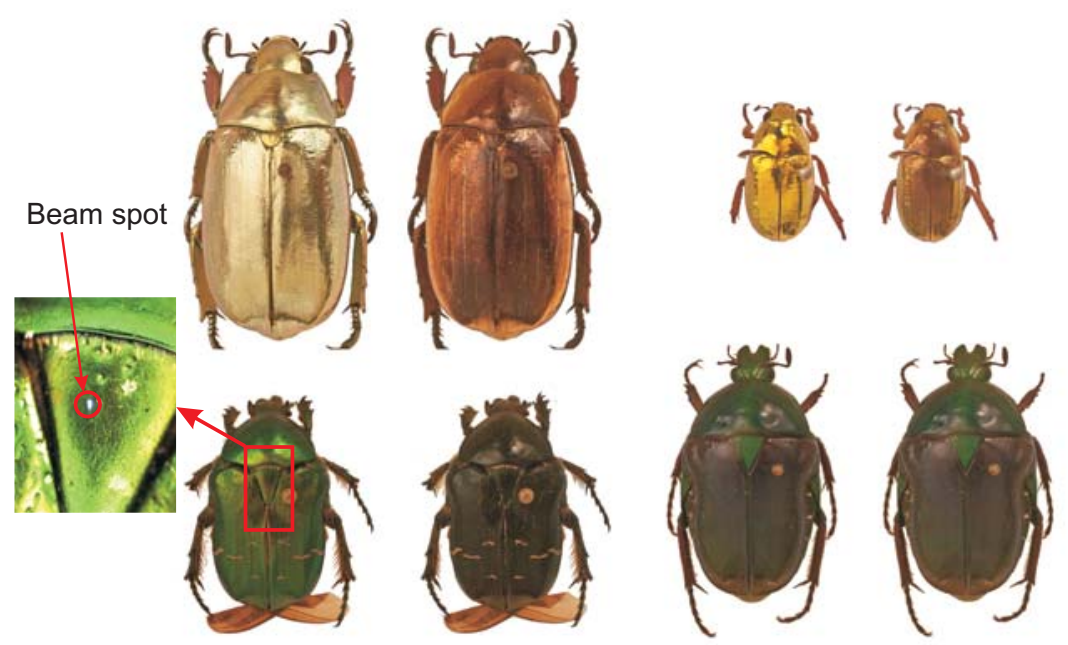

Figure 1. The four scarab beetles studied. The photos show reflection in left-handed polarized (left) and right-handed polarized (right) light. Top row: Chrysina argenteola and Anoplognathus aureus. Bottom row: Cetonia aurata and Coptomia laevis. The photo to the left shows an example of a scuttelum on which some scattered light from the beam spot can be seen as the elongated spot in the circle. (Photo Jens Birch)

The golden Anoplognathus aureus appears dark with a redbrown tone with a righthand filter and is more or less unaffected with a left-hand filter. Chrysina argenteola is also golden with some green nuances and changes its color with both right-hand and left-hand polarizing filters but does not appear black.

\section{Theory}

To facilitate the discussion of Mueller-matrix data measured on beetles, we here briefly describe the Stokes-Mueller formalism for reflection of light. In this formalism, light is described by the Stokes vector

$$
\mathbf{S}=\left[\begin{array}{l}
S_{0} \\
S_{1} \\
S_{2} \\
S_{3}
\end{array}\right]
$$

where $S_{0}=I_{x}+I_{y}, S_{1}=I_{x}-I_{y}, S_{2}=I_{+45^{\circ}}-I_{-45^{\circ}}$ and $S_{3}=I_{r}-I_{l}$. In a Cartesian $x y z$-coordinate with $z$ as the direction of propagation, $I_{x}, I_{y}, I_{+45^{\circ}}$ and $I_{-45^{\circ}}$ denote irradiances for linear polarization in the $x, y,+45^{\circ}$ and $-45^{\circ}$ directions, respectively, and $I_{r}$ and $I_{l}$ denote irradiances for right-handed and lefthanded circular polarizations, respectively.

The transformation of an input Stokes vector $\mathbf{S}_{i}$ to an output Stokes vector $\mathbf{S}_{o}$ due to interaction with a surface, e.g. an insect cuticle, is described by a Mueller matrix $\mathbf{M}$ according to

$$
\mathbf{S}_{o}=\mathbf{M S}_{i}
$$

If this equation is written out explicitly we have 


$$
\left[\begin{array}{l}
S_{o 0} \\
S_{o 1} \\
S_{o 2} \\
S_{o 3}
\end{array}\right]=\left[\begin{array}{cccc}
1 & m_{12} & m_{13} & m_{14} \\
m_{21} & m_{22} & m_{23} & m_{24} \\
m_{31} & m_{32} & m_{33} & m_{34} \\
m_{41} & m_{42} & m_{43} & m_{44}
\end{array}\right]\left[\begin{array}{c}
1 \\
S_{i 1} \\
S_{i 2} \\
S_{i 3}
\end{array}\right]
$$

where we restrict the discussion to normalized Mueller matrices $\left(m_{11}=1\right)$ and normalized Stokes vectors for the incoming light $\left(S_{i 0}=1\right)$. The normalization implies no loss of generality as we are not concerned with the absolute irradiance of reflected light, only the polarization properties.

Equation (3) can then be rewritten as

$$
\left[\begin{array}{c}
S_{o 0} \\
\mathbf{s}_{o}
\end{array}\right]=\left[\begin{array}{cc}
1 & \mathbf{D}^{T} \\
\mathbf{P} & \mathbf{m}
\end{array}\right]\left[\begin{array}{c}
1 \\
\mathbf{s}_{i}
\end{array}\right]
$$

where $\mathbf{D}=\left[m_{12}, m_{13}, m_{14}\right]^{T}$ and $\mathbf{P}=\left[m_{21}, m_{31}, m_{41}\right]^{T}$ are called diattenuation and polarizance vectors, respectively, and $\mathbf{m}$ is a $3 \times 3$ matrix. $\mathbf{s}_{i}=\left[S_{i 1}, S_{i 2}, S_{i 3}\right]^{T}$ and $\mathbf{s}_{o}=\left[S_{o 1}, S_{o 2}, S_{o 3}\right]^{T}$ are $3 \mathrm{D}$ column vectors [25]. In this report we will mainly discuss observations in unpolarized light $\left(\mathbf{s}_{i}=\mathbf{0}\right)$ and thus the polarizance $\mathbf{P}$ will be of special interest as $\mathbf{s}_{o}=\mathbf{P}$ in this case.

The Mueller matrix elements $m_{i j}$ depend on the cuticle nanostructure and a measurement of $\mathbf{M}$ allows modeling of the structure in terms of parameters like the refractive indices of cuticle materials, layer thicknesses, chirality, etc. as demonstrated in Ref. [26] and will be further presented elsewhere. However, $\mathbf{M}$ also contains all specular reflectance properties like changes in polarization and depolarization and a phenomenological description in terms of observables and derived parameters is possible. Examples of observables are $p$ - and $s$-reflectances and $p$ to $s$ mode conversion (and vice versa) where $p$ and $s$ stand for polarization parallel and perpendicular to the plane of incidence, respectively. Among the derived parameters are those of the polarization ellipse as will be described below, degree of polarization of the reflected light, color coordinates [27] and more. If the sample is isotropic and non-depolarizing, $\mathbf{M}$ also contains the ellipsometric parameters $\Psi$ and $\Delta$ defined by $\rho=r_{p} / r_{s}=\tan \Psi e^{i \Delta}$, where $r_{p}$ and $r_{s}$ are the reflection coefficients for $p$ and $s$-polarization, respectively. Specifically for an isotropic and non-depolarizing surface it holds that $m_{22}=1,-m_{12}=-m_{21}=\cos 2 \Psi, m_{33}=m_{44}=\sin 2 \Psi \cos \Delta$, $m_{34}=-m_{43}=\sin 2 \Psi \sin \Delta$ and with all remaining elements equal to zero [28].

It is of interest to understand the significance of the various elements $m_{i j}$. Values close to zero on $m_{34}$ and $m_{43}$ together with $m_{12}$ and $m_{21}$ not close to one implies that $\sin \Delta$ is small, i.e. $\Delta$ is small which is indicative of a dielectric mirror as will be the case in some spectral regions for the beetles. The elements $m_{41}$ and $m_{14}$ are of special interest. Negative values correspond to left-handed and positive values to right-handed polarization of the reflected light [29]. In the special case when $m_{21}=m_{31}=0$ and $m_{41} \neq 0$, a surface reflects circularly polarized light for incident unpolarized light. This is immediately seen if $\mathbf{S}_{i}=[1,0,0,0]^{T}$ is used in Equation (2) whereby $\mathbf{S}_{o}=\left[1,0,0, m_{41}\right]^{T}$, i.e. the reflected light is circularly polarized. If $m_{21} \neq 0$ and/or $m_{31} \neq 0$, the reflected light will instead be elliptically polarized.

The state of polarization of light can be visualized with the polarization ellipse as illustrated in Figure 2. In an $x y z$ coordinate system with $z$ as the direction of light propagation, the polarization ellipse is simply the path traced out by the electric field of polarized light at a fixed $x y$-plane. Two parameters are needed to 


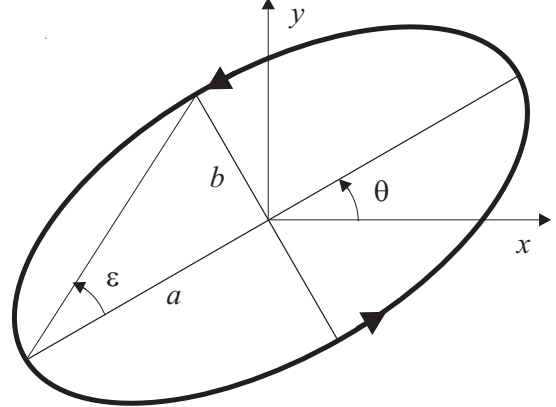

Figure 2. The polarization ellipse for visualization of the state of polarization using the convention of looking into the beam. In the example shown the electric field vector rotates anticlockwise (left-handed polarization) as shown by the arrows on the ellipse.

describe polarization: the azimuth angle $\theta$ which is the angle between the major axis and the $x$-axis and the ellipticity $e=b / a$ which is the ratio between the length of the minor axis $b$ and the major axis $a$. We will also use the ellipticity angle $\epsilon=\arctan e$. The handedness is included as the sign of $e$, or alternatively $\epsilon$, and is negative (positive) for left(right)-handed polarization. The parameters $\theta$ and $\epsilon$ are related to a Stokes vector $\mathbf{S}$ as [28]

$$
\begin{gathered}
\epsilon=\frac{1}{2} \arcsin \frac{S_{3}}{\sqrt{S_{1}^{2}+S_{2}^{2}+S_{3}^{2}}} \\
\theta=\frac{1}{2} \arctan \left(S_{2} / S_{1}\right)
\end{gathered}
$$

For the special case of incident unpolarized light $\left(\mathbf{s}_{i}=0\right)$, we can calculate $\mathbf{s}_{o}$ from Equation (4). Equations (5) and (6) then reduce to

$$
\begin{gathered}
\epsilon=\frac{1}{2} \arcsin \frac{m_{41}}{\sqrt{m_{21}^{2}+m_{31}^{2}+m_{41}^{2}}} \\
\theta=\frac{1}{2} \arctan \left(m_{21} / m_{31}\right)
\end{gathered}
$$

The degree of polarization $P$, will also be used. If only the part $I_{\text {pol }}$ of the total irradiance $I_{\text {tot }}$ is polarized, $P$ is defined as $P=I_{\text {pol }} / I_{\text {tot }}=\sqrt{S_{1}^{2}+S_{2}^{2}+S_{3}^{2}} / S_{0}[28]$. If light incident to a surface is unpolarized, i.e. $\mathbf{s}_{i}=0$, we obtain for the reflected light

$$
P=\sqrt{m_{21}^{2}+m_{31}^{2}+m_{41}^{2}}
$$

We also observe that $P=|\mathbf{P}|$ and that $m_{41}=P \sin 2 \epsilon$.

It should be pointed out that $P_{\mathbf{s}_{i}=0}$ in the special case when $m_{21}=m_{31}=0$, i.e. circularly polarized light, reduces to the degree of polarization introduced by Pye, i.e. $(1-\alpha) /(1+\alpha) \rightarrow\left|m_{41}\right|$. The description in Ref. [9] is thus incomplete as it is well defined only for circular polarization and physically corresponds to the degree of circular polarization produced by unpolarized light, whereas $m_{21}$ and $m_{31}$ correspond to the horizontal and $45^{\circ}$-linear degree of polarization, respectively [30]. 
As an example, linearly polarized light has $\alpha=0$ and thereby will appear as having zero degree of polarization in Pye's formalism and for elliptically polarized light, only the circular part contributes. Furthermore $\alpha$ is a broadband parameter and in a measurement an integration is made over the spectral range of the left-hand and right-hand polarizing filters used. As a consequence a surface which reflects left-handed polarization in one part of the spectral range may be counteracted by right-handed polarization in another part (such examples will be given later in the report). In a worst case scenario these two contributions may cancel each other and the surface will appear as if there is no circular polarization at all. We therefore strongly recommend the use of the definition in Equation (9).

\section{Experimental}

Mueller-matrix data were recorded with precision better than \pm 0.005 in the Mueller-elements $m_{i j}$ using a dual rotating compensator ellipsometer (RC2, J. A. Woollam, Co., Inc.). A full description of this type of instrument is given by Collins and Koh [31]. The spectral range was $245-1700 \mathrm{~nm}$ and angles of incidence $\phi$ in the range $20^{\circ}-75^{\circ}$ measured from the surface normal were used. With focusing optics, an elliptically shaped beam spot with size $50 \times 50(\cos \phi)^{-1} \mu \mathrm{m}$ was obtained. All measurements were performed on the scutellum, which is a small triangular plate on the dorsal side on the investigated beetles (see Figure 1). The instrument is equipped with a camera integrated with the ellipsometer software to facilitate positioning and alignment of samples. A motorized xy-stage allows positioning of the light spot with a resolution of $1 \mu \mathrm{m}$ making it possible to select a flat region free from protrusions and ridges. An alignment detector makes it possible to adjust tilt and z-translation to assure that the sample is accurately aligned. Only data in the spectral range $300-900 \mathrm{~nm}$ are reported in this investigation. Calculations of the derived parameters azimuth $\theta$, ellipticity $\epsilon$ and degree of polarization $P$ were done with the software CompleteEASE (J. A. Woollam, Co., Inc.).

\section{Results and discussion}

First we present Mueller matrices for the four beetles studied. The variation with angle of incidence and wavelength is high-lighted and we demonstrate how a complete description is provided with a so called contour plot. We then discuss leftand right-handedness in the polarization of reflected light. Finally a few examples on derived parameters are presented.

\subsection{Mueller-matrices for the four beetles}

Figure 3 shows Mueller-matrix spectra for Cetonia aurata measured at an angle of incidence of $20^{\circ}$. For wavelengths below $500 \mathrm{~nm}$ and above $650 \mathrm{~nm}$, all Mueller matrix elements are more or less constant and the cuticle structure optically can be considered as a dielectric surface. Accordingly $m_{22}$ is close to unity and the elements representing - $\cos 2 \Psi$, i.e. $m_{12}$ and $m_{21}$, are identical within instrumental resolution and with values around -0.17 . Furthermore, $m_{34}$ and $m_{43}(\sin 2 \Psi \sin \Delta)$ are close to zero implying $\Delta \approx 0 . m_{33}$ as well as $m_{44}$ represent $\sin 2 \Psi \cos \Delta$ and are close to -1 which is in accordance with the values of $m_{12}$ and $m_{21}$. All remaining elements are close to zero.

In the spectral region 500-650 $\mathrm{nm}$, all elements show some spectral variation. In particular we notice that $m_{14}=m_{41}<0$. Hence, incoming unpolarized light is 


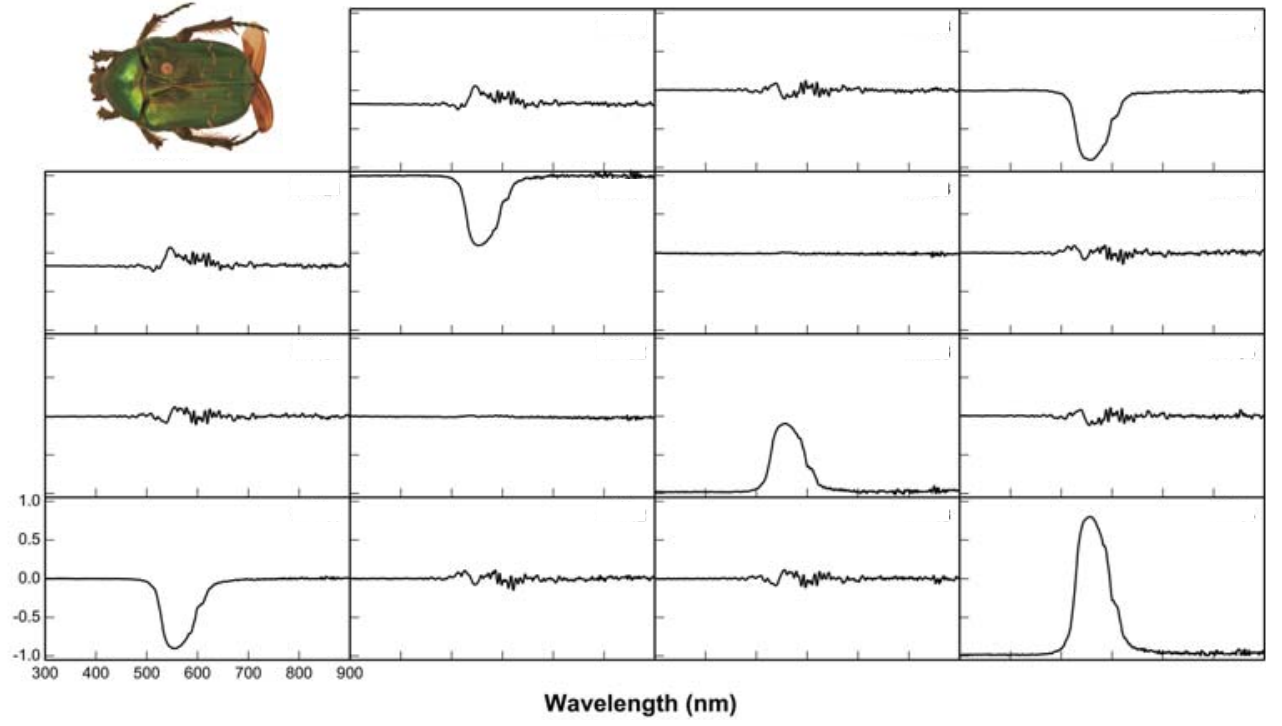

Figure 3. Normalized Mueller-matrix spectra for Cetonia aurata at an angle of incidence of $20^{\circ}$. The position of each panel corresponds to the element at the corresponding position in the Mueller matrix in Equation (3). The scales are the same for all panels and are shown for the panel in the lower left corner (element $\left.m_{41}\right)$.

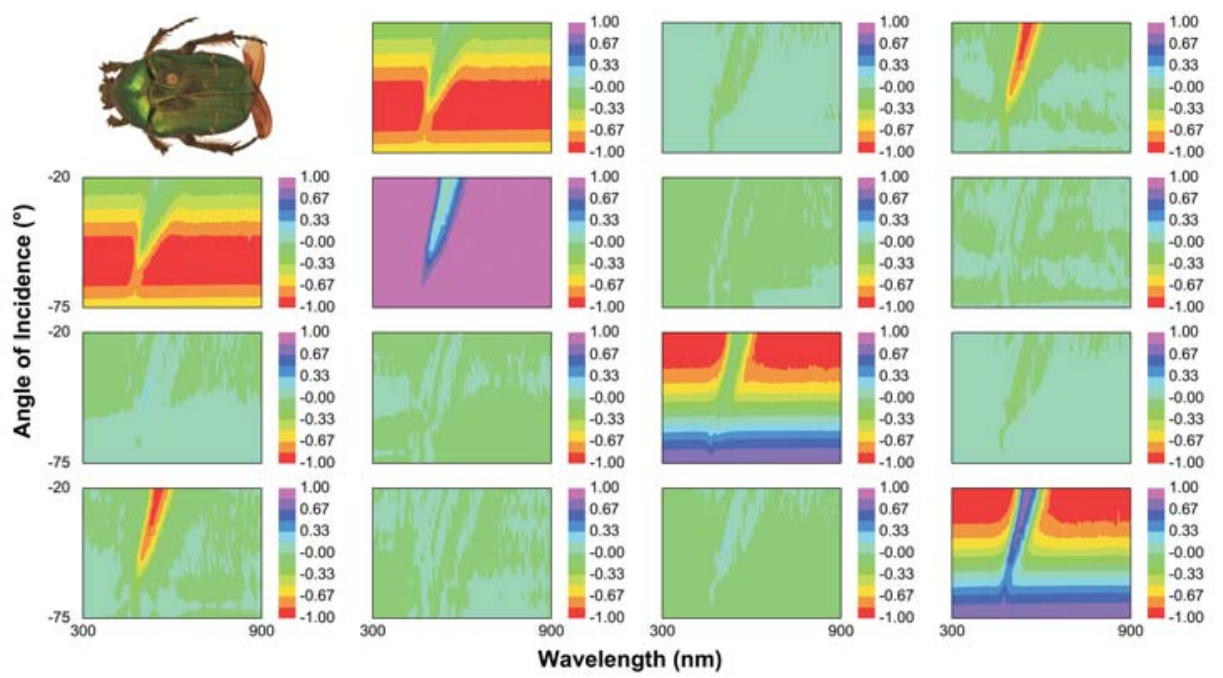

Figure 4. Contour plots of Mueller-matrix elements for Cetonia aurata at angles of incidence between $20^{\circ}$ and $75^{\circ}$.

reflected with left-handed polarization. According to Equation (7) the polarization is elliptical, not circular, as both $m_{21}$ and $m_{31}$ are non-zero. Additional symmetry properties are $m_{21}=m_{12}$ and $m_{34}=-m_{43}$. This beetle is green at normal incidence but with the naked eye, it can be seen that the beetle color changes from green to blue when the viewing angle increases. This is also evident from the contour plot in Figure 4, which presents spectral measurements in the angle of incidence range $20^{\circ}$ to $75^{\circ}$. Furthermore it is seen that the left-handed effect decreases with increasing angle of incidence, e.g. in $m_{41}$ which approaches zero at large angles.

Figure 5 shows (on an expanded scale) ellipsometrically determined Muellermatrix spectra for Coptomia laevis measured at an angle of incidence of $45^{\circ}$. It is seen that $m_{13}, m_{14}, m_{23}, m_{24}, m_{31}, m_{32}, m_{34}, m_{41}, m_{42}$ and $m_{43}$ are close to zero $(<0.05) \cdot m_{14}$ and $m_{41}$ show some very minute left-handed effects around $500 \mathrm{~nm}$. The fact that $m_{34}$ and $m_{43}$ are very small implies a dielectric surface with $\Delta \approx 0$.

From the Mueller-matrix data the ellipsometric parameters $\Psi$ and $\Delta$ can be 


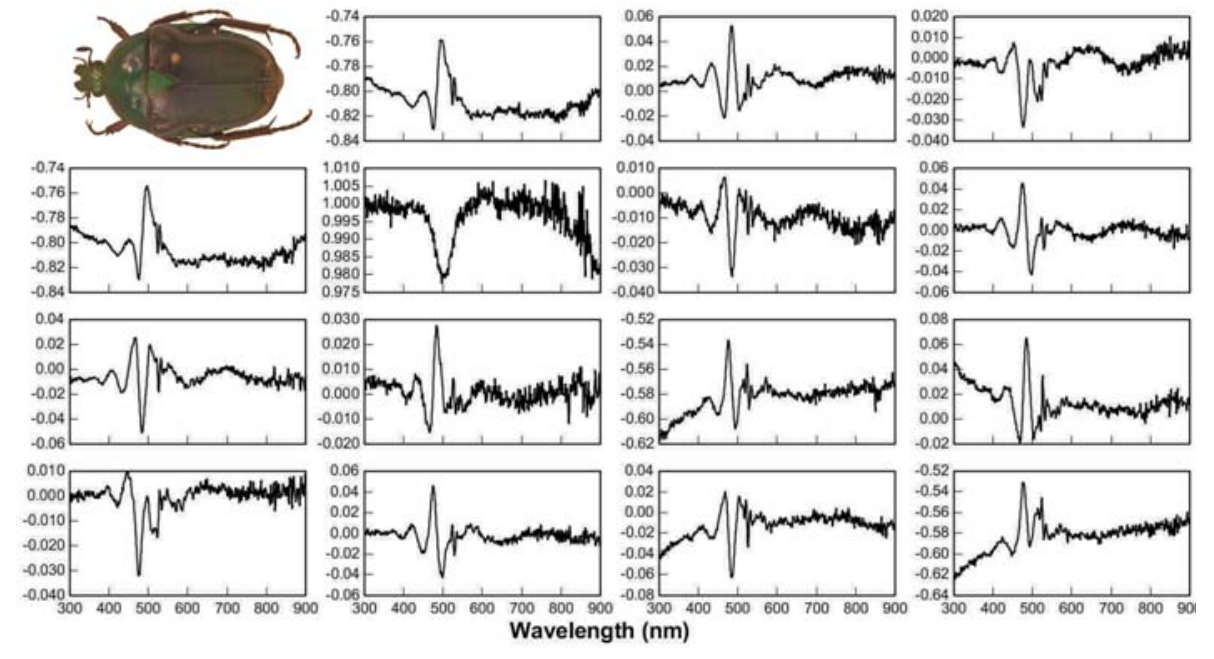

Figure 5. Mueller-matrix spectra for Coptomia laevis at an angle of incidence of $45^{\circ}$. Observe that the vertical axes are expanded and different for the different elements to facilitate comparison among the elements.

determined [28] and the pseudo-refractive index $\langle N\rangle=\langle n\rangle+i\langle k\rangle$ is obtained by inserting $\rho=\tan \Psi e^{i \Delta}$ in

$$
<N>=<n>+i<k>=\sin \phi \sqrt{1+\left(\frac{1-\rho}{1+\rho}\right)^{2} \tan ^{2} \phi}
$$

Except for a narrow region around $500 \mathrm{~nm},\langle N\rangle$ is similar for different angles of incidence. The value of $\langle n\rangle$ is in the range 1.54 to 1.56 with the larger value at shorter wavelengths and $\langle k\rangle$ is 0.03 or smaller. These values are typical for dielectric organic materials [32], [33]. So, although the actual nanostructure of the cuticle is very complex, an equivalent index of the cuticle material can be estimated.

Figures 6 and 7 show Mueller-matrix spectra for Chrysina argenteola and Anoplognathus aureus measured at an angle of incidence of $45^{\circ}$. We observe that these beetles have broad-band features which are more complex than for $\mathrm{Ce}$ tonia aurata. Both Chrysina argenteola and Anoplognathus aureus exhibit lefthandedness at this angle of incidence and interference oscillations are clearly seen for wavelengths larger than $500 \mathrm{~nm}$. These oscillations are very weak in Cetonia aurata and Coptomia laevis. The interference oscillations carry information about the thickness of the color generating parts of the cuticle and will be further evaluated in future communications.

\subsection{Left- and right-handedness deduced from $m_{41}$}

In Figure 8, $m_{41}$ spectra are shown for the four beetles. The narrow band reflection for the green Cetonia aurata is noticable. Coptomia laevis exhibits minor elliptical polarization effects and has virtually zero $m_{41}$ compared to the other beetles and its green color is most probably due to ordinary thin film interference in a multilayer stack. From an optical point of view Coptomia laevis appears as a dielectric mirror over the entire spectral range. Anoplognathus aureus has a color very similar to that of gold and exhibits a negative $m_{41}$ for wavelengths larger than $480 \mathrm{~nm}$ as seen in Figure 8. Chrysina argenteola also appears golden to the eye but to a lower 


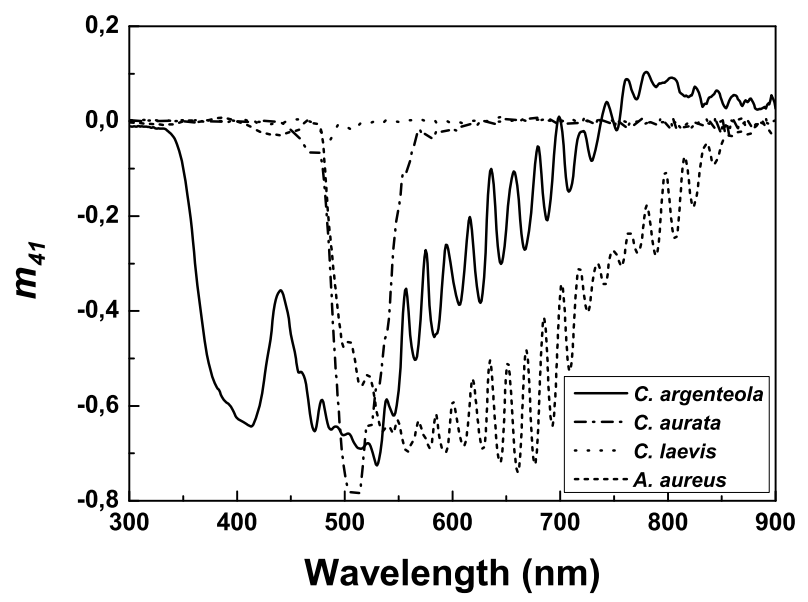

Figure 8. Mueller-matrix element $m_{41}$ for Chrysina argenteola, Cetonia aurata, Coptomia laevis and Anoplognathus aureus at an angle of incidence of $45^{\circ}$.

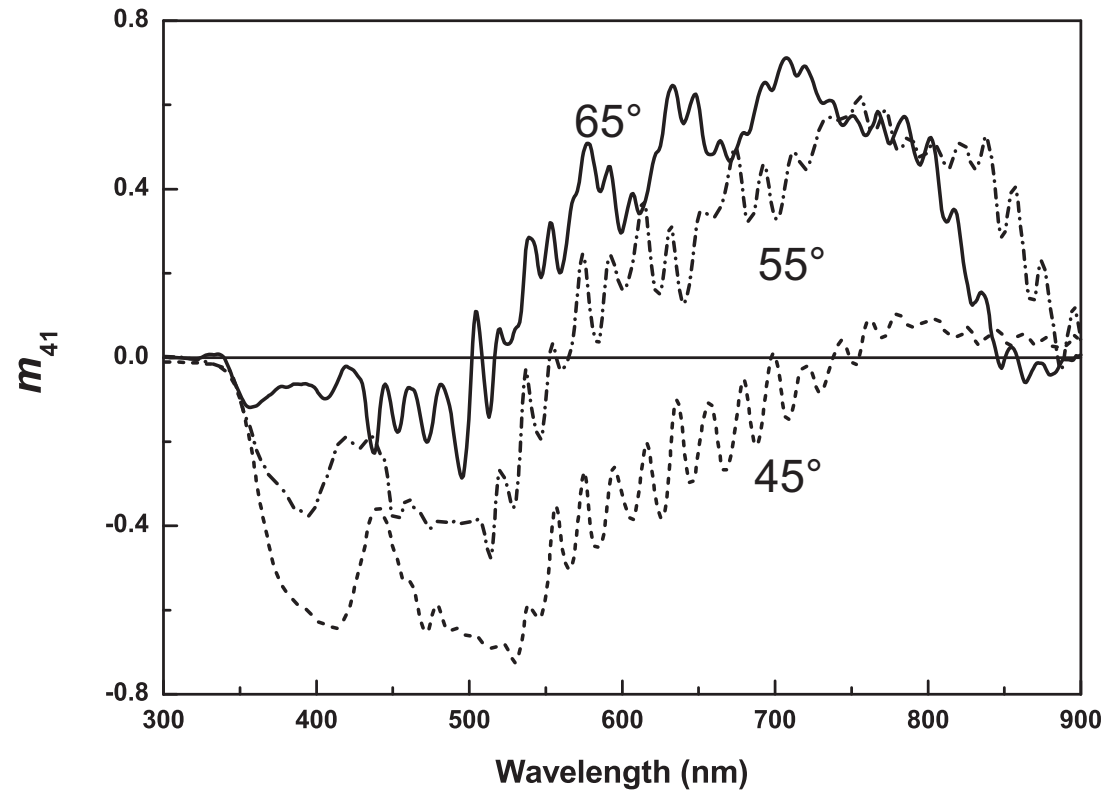

Figure 9. Mueller-matrix element $m_{41}$ for Chrysina argenteola at angles of incidence of $45^{\circ}, 55^{\circ}$ and $65^{\circ}$.

$m_{31}$ are small and approach $e=1\left(\epsilon=45^{\circ}\right)$ when both $m_{21}$ and $m_{31}$ approach zero. However, this is not a sufficient condition to generate reflected light which is completely circularly polarized. This is correct if only the polarized part of the light is considered but in a more complete description it is also of importance to consider how much of the reflected light that in fact is polarized, i.e. to determine $P$. It is seen in Equation (7), that even with $m_{41}$ very small, we can have $e$ close to one, i.e. circularly polarized light, in the case when $m_{21}=m_{31}=0$. To have a substantial part of the reflected light really being circular, $m_{41}$ should not be too small in addition to $m_{21}=m_{31}=0$. This can be quantified if the degree of polarization $P=|\mathbf{P}|$ is determined from Equation (9) where it can be seen that totally polarized circularly polarized light $\left(m_{21}=m_{31}=0\right)$ requires $m_{41}=1$. Figure 11 illustrates the degree of polarization $P$ at an angle of incidence of $45^{\circ}$ calculated from Equation (9) using $m_{41}$ data and the corresponding $m_{21}$ and $m_{31}$ data for the four scarab beetles studied. Notice that $P$ is 0.5 or larger for most wavelengths for all four beetles studied. The azimuth $\theta$, the tilt of the polarization 


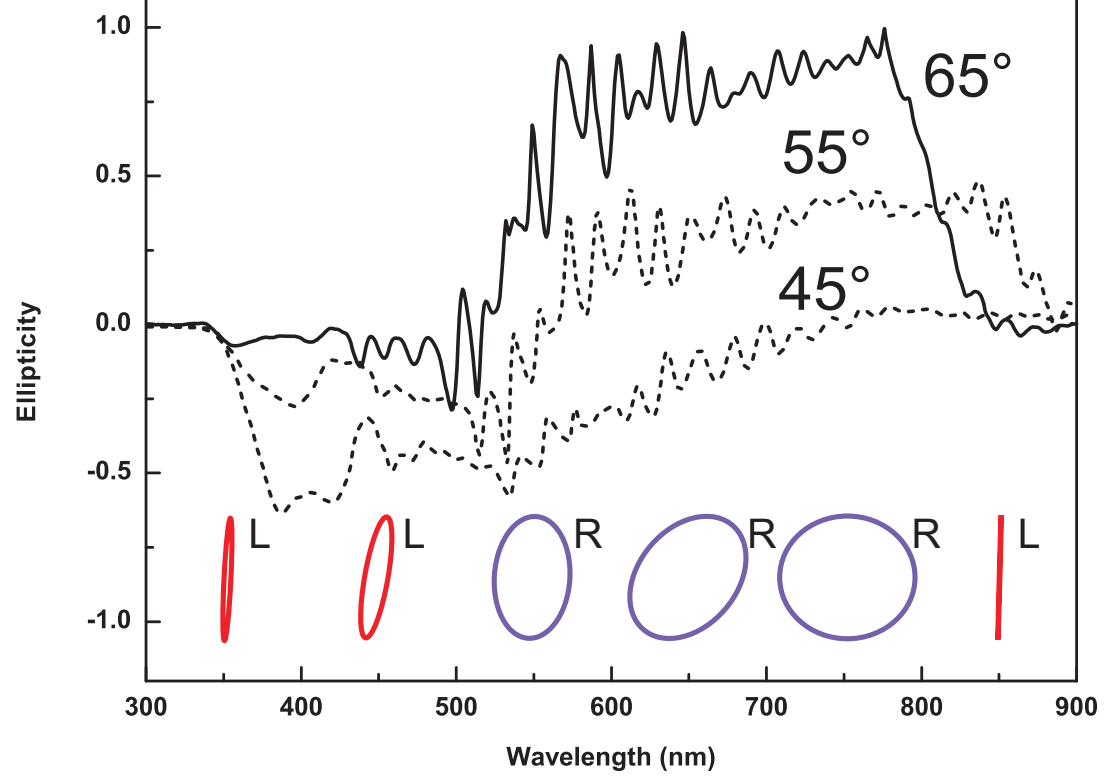

Figure 10. Ellipticity $e$ of light reflected from Chrysina argenteola for incident unpolarized light at angles of incidence of $45^{\circ}, 55^{\circ}$ and $65^{\circ}$. The insets illustrate the state of polarization at selected wavelengths and at $65^{\circ}$ with $\mathrm{L}(\mathrm{R})$ indicating left-handed (right-handed) polarization.

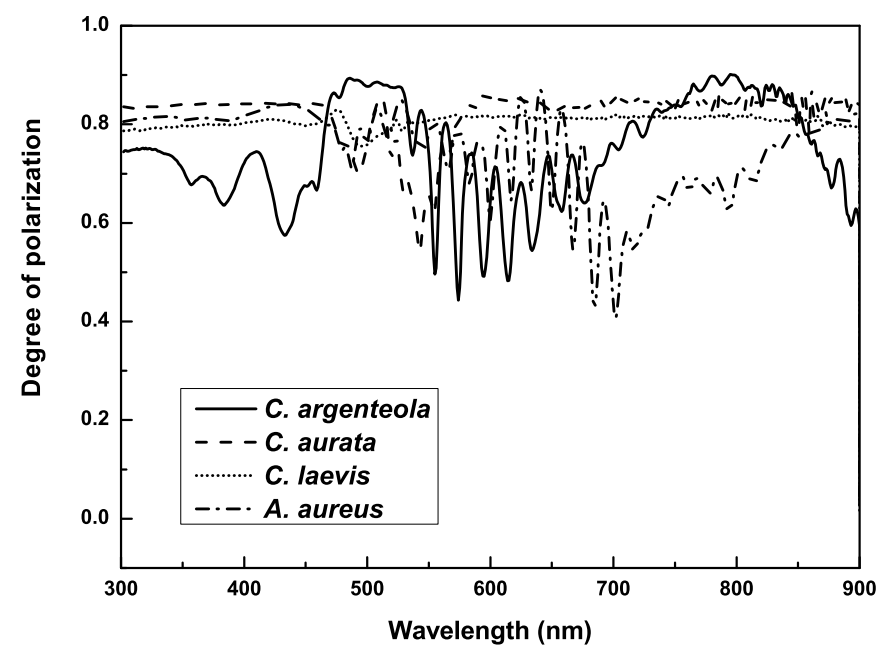

Figure 11. Degree of polarization of light reflected at an angle of incidence of $45^{\circ}$ for the four scarab beetles studied.

ellipse, is a parameter which can be determined using Equation (8) but is not shown here.

\subsection{Biological significance}

Very little is known about the biological significance of the polarizing phenomena studied here. According to Pye [9] it is found only in scarabaeoids and he suggests that the eyes of these beetles can detect circular polarization. Such capabilities are known from stomatopod crustaceans [34] and has also recently been observed by Brady and Cummings in Chrysina gloriosa [35] which can discriminate circular polarization from linear polarization as well as from unpolarized light. The fact that this beetle also exhibits circularly polarized reflections indicates that circular 
polarization vision is important from an ecological point of view, possibly for intraspecies communication. Even if a beetle, like Chrysina gloriosa, may be able to discriminate between polarized and unpolarized light, it is not given that the degree of polarization matters as it may correlate with the irradiance in case the beetle is blind to unpolarized light. It therefore seems appropriate to further investigate the role of degree of polarization in future work.

The superfamily Scarabaeoidea contains approximately 35000 species worldwide, grouped in approximately 12 families. Pye [9], with a simpler technique than ellipsometry, found many species exhibiting polarizing properties in 8 of these families, including species studied by earlier authors. Only Hodgkinson et al. [16] and we have used modern ellipsometry, allowing thorough description and analysis of the structure of the cuticle, to be presented. The number of studied species still is only 8 , representing 2 or 3 of the families. These 8 species vary much in polarizing properties. Accordingly several more species and families should preferrably be studied to learn and generalize about polarizing properties and cuticle structure in the Scarabaeoidea. In conclusion it is fair to say that the research about polarized reflection in beetles still is in its infancy.

\section{Summary of findings}

Normalized Mueller-matrix spectra at oblique incidence, of a quality not hitherto available, are presented for a selection of scarab beetles. Our main findings are:

- Mueller matrices are very rich in information about reflection properties of scarab beetles and the existence of right-handedness in polarization is clarified.

- The concept of polarization in the reflection from beetles is generalized from a simple classification in circular or not circular to a general description of elliptical polarization including linear and circular polarization as special cases. The elliptical polarization is quantified in azimuth and ellipticity of the polarization ellipse.

- It is confirmed that both left- and right-handed polarization can be found in light reflected from the same beetle depending on wavelength. We also show that leftand right-handed polarization can be observed at the same wavelength but at different angles of incidence.

- The degree of polarization may be of importance and we recommend that this is included in a more complete description of cuticle reflection even though there is no evidence so far of its relevance in biology.

In future work Mueller-matrix data will help us to understand the nanostructure of beetle cuticles and will provide knowledge of polarization coding in reflection from beetles. The latter will be crucial for understanding polarization-related ecological questions.

\section{Acknowledgements}

Financial support was obtained from the Knut and Alice Wallenberg foundation and the Swedish Research Council. The Museum of Natural History in Stockholm is acknowledged for loan of beetles. Jens Birch is acknowledged for providing beetle images. 
[1] A.A. Michelson, Phil. Mag. 21 (1911) p.554-567.

[2] C. Robinson, Molecular Crystals 1 (1966) p.467-494.

[3] A.C. Neville and S. Caveney, Biol. Rev. 44 (1969) p.531-562.

[4] Y. Bouligand, J. Phys. Colloques 30 (1969) p.C4-90-C4-103.

[5] A.E. Seago, P. Brady, J.P. Vigneron and T.D. Schultz, J. R. Soc. Interface 6 (2009) p.S165-S184.

[6] T. Lenau and M. Barfoed, Adv. Eng. Mat. 10 (2008) p.299-314.

[7] P. Vukusic and J.R. Sambles, Nature 424 (2003) p.852-855.

[8] S. Kinoshita Structural colors in the realm of nature, World-Scientific, 2008.

[9] J.D. Pye, Biol. J. Linnean Soc. 100 (2010) p.585-596.

[10] A.B.T. Smith, D.C. Hawkins and J.M. Heraty, Coleopterists Soc. Monograph 5 (2006) p.35-46.

[11] A.B.T. Smith, Coleopterists Soc. Monograph 5 (2006) p.144-204.

[12] S. Berthier, E. Charron and J. Boulenguez, Insect Science 13 (2006) p.3-12.

[13] S. Caveney, Proc. Roy. Soc. Lond. B. 178 (1971) p.205-224.

[14] S. Lowrey, L.D. Silva, I. Hodgkinson and J. Leader, J. Opt. Soc. Am. A 24 (2007) p.2418-2425.

[15] L.D. Silva, I. Hodgkinson, P. Murray, Q.H. Wu, M. Arnold, J. Leader and A. McNaughton, Electromagnetics 25 (2005) p.391-408.

[16] I. Hodgkinson, S. Lowrey, L. Bourke, A. Parker and M.W. McCall, Appl. Opt. 49 (2010) p.4558-4567.

[17] S. Berthier, J. Boulenguez and Z. Bálint, Appl. Phys. A 86 (2007) p.123-130.

[18] R.A. Potyrailo, H. Ghiradella, J.R.C. A. Vertiatchikh K. Dovidenko and E. Olson, Nature Photonics 1 (2007) p.123-128.

[19] K. Watanabe, T. Hoshino, K. Kanda, Y. Haruyama and S. Matsui, Jpn. J. Appl. Phys. 44 (2005) p.L48-L50.

[20] A.R. Parker and H.E. Townley, Nature Nanotechnology 2 (2007) p.347-351.

[21] R. Hegedüs, G. Szél and G. Horváth, Vision Research 46 (2006) p.2786-2797.

[22] H.G. Tompkins and E.A. Irene Handbook of Ellipsometry, William Andrew publishing, 2005.

[23] D.J. Brink and M.E. Lee, Appl. Opt. 35 (1996) p.1950-1955.

[24] D.H. Goldstein, Appl. Opt. 45 (2006) p.7944-7950.

[25] S. Lu and R.A. Chipman, Opt. Comm. 146 (1998) p.11014.

[26] H. Arwin, Thin Solid Films 519 (2011) p.2589-2592.

[27] B. Johs, H. Arwin, T. Wagner, D. Appel and D. Peros, Thin Solid Films 519 (2011) p.2711-2714.

[28] R.M.A. Azzam and N.M. Bashara Ellipsometry and Polarized Light, North-Holland, 1986.

[29] E. Georgieva, Appl. Opt. 30 (1991) p.5081-5085.

[30] S. Lu and R.A. Chipman, J. Opt. Soc. Am. A 13 (1996) p.1106-1113.

[31] R.W. Collins and J. Koh, J. Opt. Soc. Am. A 16 (1999) p.1997-2006.

[32] H. Arwin, Appl. Spec. 40 (1986) p.313-318.

[33] S. Berthier, E. Charron and A.D. Silva, Opt. Comm. 228 (2003) p.349-356.

[34] T.H. Chiou, S. Kleinlogel, T. Cronin, R. Caldwell, B. Loeffler, A. Siddigi, A. Goldizen and J. Marshall, Curr. Biol. 18 (2008) p.429-434.

[35] P. Brady and M. Cummings, The Amer. Naturalist 175 (2010) p.614-620. 